\title{
High accuracy trigonometric approximations of the real Bessel functions of the first kind
}

\author{
Annie Cuyt ${ }^{1}$, Wen-shin $\mathrm{Lee}^{1,2}$, and Min $\mathrm{Wu}^{3 \star}$ \\ ${ }^{1}$ Universiteit Antwerpen, Dept. of Mathematics and Computer Science, \\ Middelheimlaan 1, B-2020 Antwerpen, Belgium \\ annie. cuyt@uantwerpen. be \\ ${ }^{2}$ University of Stirling, Computing Science and Mathematics, Stirling FK9 4LA, \\ Scotland, UK \\ wen-shin.lee@stir.ac.uk \\ ${ }^{3}$ East China Normal University, School of Computer Science and Software \\ Engineering, Shanghai Key Laboratory of Trustworthy Computing, Shanghai 200062, \\ P.R. China \\ mwui@sei.ecnu.edu.cn
}

\begin{abstract}
We construct high accuracy trigonometric interpolants from equidistant evaluations of the Bessel functions $J_{n}(x)$ of the first kind and integer order. The trigonometric models are cosine or sine based depending on whether the Bessel function is even or odd. The main novelty lies in the fact that the frequencies in the trigonometric terms modelling $J_{n}(x)$ are also computed from the data in a Prony-type approach. Hence the interpolation problem is a nonlinear problem. Some existing compact trigonometric models for the Bessel functions $J_{n}(x)$ are hereby rediscovered and generalized.
\end{abstract}

\section{Bessel functions of the first kind}

The Bessel functions $J_{\nu}(x)$ of the first kind and order $\nu$ satisfy the second order differential equation

$$
x^{2} \frac{d^{2} y(x)}{d x^{2}}+x \frac{d y(x)}{d x}+\left(x^{2}-\nu^{2}\right) y(x)=0, \quad \nu \in \mathbb{C} .
$$

They are therefore especially important in many scientific computing problems involving wave propagation and static potentials. Among others, we mention signal processing, electromagnetics, acoustical radiation and vibration analysis.

Solving problems in cylindrical coordinate systems leads to Bessel functions of integer order, while in spherical problems, one obtains half-integer orders. So the functions $J_{\nu}(x)$ of integer and half integer order, where respectively $\nu=n \in \mathbb{Z}$ and $\nu=(2 n+1) / 2$ are among the more important. We are interested in Bessel

\footnotetext{
* This research was carried out while Min Wu was taking up a 1-year research grant supported by the Universiteit Antwerpen (Belgium)
} 
functions of the first kind of positive integer order and real arguments. Note that for negative integer order we have the relation

$$
J_{-n}(x)=(-1)^{n} J_{n}(x)=J_{n}(-x), \quad n \in \mathbb{N}, \quad x \in \mathbb{R} .
$$

The even and odd behaviour of the functions $J_{n}(x)$ is illustrated in the graphs of $J_{0}(x)$ and $J_{1}(x)$ shown in Figure 1.
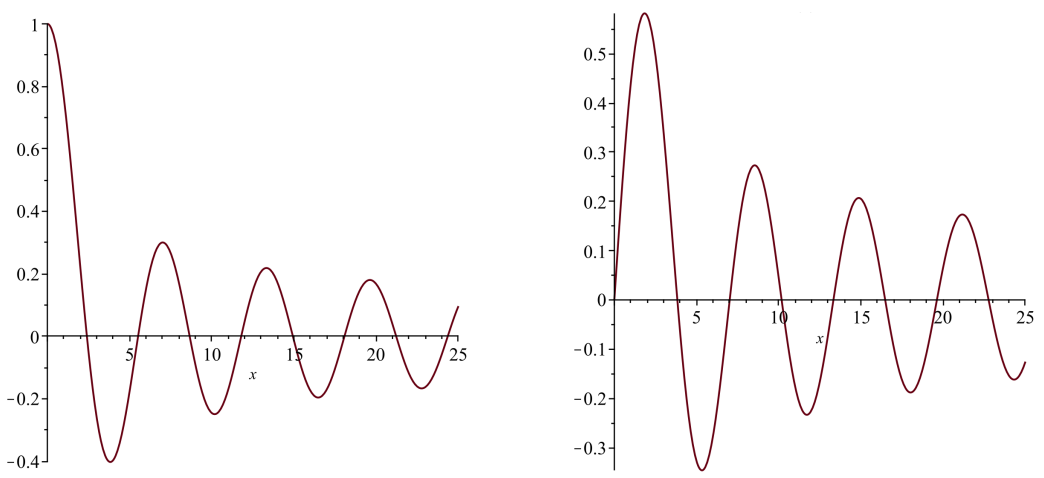

Fig. 1. The graphs of $J_{0}(x)$ (left) and $J_{1}(x)$ (right).

The power series and asymptotic series expansions of $J_{n}(x)$ are respectively given by

$$
J_{n}(x)=\sum_{k=0}^{\infty} \frac{(-1)^{k}}{k !(n+k) !}\left(\frac{x}{2}\right)^{2 k+n}, \quad x \geq 0
$$

and

$$
\begin{aligned}
& J_{n}(x)= \sqrt{\frac{2}{\pi x}}\left(\cos \left(x-(n+1 / 2)^{\pi / 2}\right) \sum_{k=0}^{\infty}(-1)^{k} \frac{\Gamma(1 / 2+2 k+n)}{k ! \Gamma(1 / 2-2 k+n)(2 x)^{2 k}}+\right. \\
&\left.-\sin \left(x-(n+1 / 2)^{\pi} / 2\right) \sum_{k=0}^{\infty}(-1)^{k} \frac{\Gamma(1 / 2+2 k+n+1)}{k ! \Gamma(1 / 2-2 k+n-1)(2 x)^{2 k+1}}\right), \\
& x \rightarrow \infty
\end{aligned}
$$

where for $\ell \in \mathbb{N}$,

$$
\begin{aligned}
& \Gamma(1 / 2+\ell)=\frac{(2 \ell-1) ! !}{2^{\ell}} \sqrt{\pi}, \\
& \Gamma(1 / 2-\ell)=\frac{(-2)^{\ell}}{(2 \ell-1) ! !} \sqrt{\pi} .
\end{aligned}
$$

In the literature, various trigonometric approximations of the Bessel functions $J_{n}(x)$ can be found, including Fourier series expansions and quadrature formulas 
applied to integral representations. Mostly these approximations are constructed to provide simple compact models guaranteeing a few significant digits on a finite interval $0 \leq x \leq B$. For instance, in [2] we find

$$
\begin{aligned}
& J_{0}(x)=\frac{1}{6}+\frac{1}{3} \cos (x / 2)+\frac{1}{3} \cos (\sqrt{3} x / 2)+\frac{1}{6} \cos (x), \\
& J_{4}(x)=\frac{1}{8}-\frac{1}{4} \cos (\sqrt{2} x / 2)+\frac{1}{8} \cos (x) .
\end{aligned}
$$

Our aim is to provide equally compact formulas constructed from discrete data, using suitably selected frequencies and delivering a high accuracy approximant in $0 \leq x \leq B$.

\section{Trigonometric approximations}

From the graphs of the Bessel functions $J_{n}(x)$ of the first kind for integer orders, one notices that they behave very much like decaying trigonometric functions. Also, through the reflection formula

$$
J_{n}(-x)=(-1)^{n} J_{n}(x), \quad x \geq 0,
$$

and the fact that $J_{0}^{\prime}(x)=-J_{1}(x)$, the behaviour is similar to that of the cosine and sine functions.

\subsection{Finite real argument restriction}

Since our trigonometric approximations are constructed from a finite number of uniformly collected samples of $J_{n}(x)$, we focus on the behaviour of $J_{n}(x)$ and its approximations on a finite interval $[0, B]$ with $B>0$. We therefore also introduce the functions

$$
\begin{aligned}
J_{0}(B ; x) & :=J_{0}(x), \quad 0 \leq x \leq B, \\
J_{n}(B ; x) & :=(B / x) J_{n}(x), \quad 0<x \leq B,
\end{aligned}
$$

for some specific restriction of the real Bessel function $J_{n}(x)$ to the finite interval $[0, B]$. This restriction doesn't inherently change the behaviour of the Bessel function, as we illustrate in Figure 2 where we display $J_{2}(25 ; x)$ : with $B / x \geq 1$ for $0<x \leq B$ the function $J_{n}(B ; x)$ is a somewhat magnified version of $J_{n}(x)$.

For increasing $B$ we find that the new approximations introduced in this section, have a far better overall behaviour, meaning a smaller uniform norm of the relative error, than the existing power series and asymptotic series expansions which only perform well at either end of the interval $[7,3]$. 


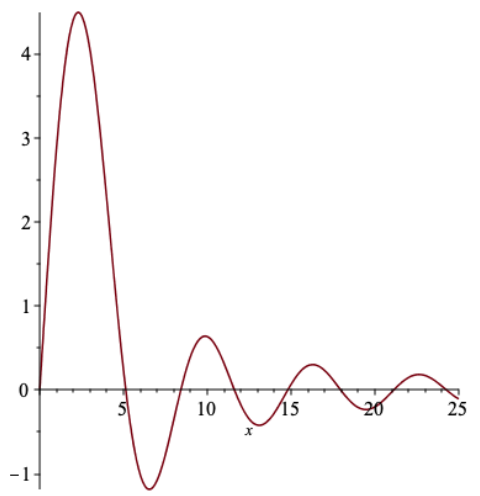

Fig. 2. The graph of $J_{2}(25 ; x)$.

In the numerical illustrations we also compare to partial sums of the Fouriertype series representations [1]

$$
\begin{gathered}
J_{0}(B ; x)=\sum_{k=0}^{\infty} \delta_{k} \frac{2}{\sqrt{B^{2}+k^{2} \pi^{2}}} \sin \left(\sqrt{B^{2}+k^{2} \pi^{2}}\right) \cos \left(k \pi \sqrt{1-x^{2} / B^{2}}\right) \\
J_{n}(B ; x)=\sum_{k=0}^{\infty} \delta_{k} \pi J_{\frac{n}{2}}\left(\frac{\sqrt{B^{2}+k^{2} \pi^{2}}+k \pi}{2}\right) J_{\frac{n}{2}}\left(\frac{\sqrt{B^{2}+k^{2} \pi^{2}}-k \pi}{2}\right) \\
\times \cos \left(k \pi \sqrt{1-x^{2} / B^{2}}\right), \quad n \geq 1
\end{gathered}
$$

where $\delta_{0}=1 / 2$ and $\delta_{k}=1, k>0$.

From (4) and the definitions (5) it is clear that for $n \geq 0$ the $J_{2 n}(x)$ and the $J_{2 n+1}(B ; x)$ are even functions and that the $J_{2 n+1}(x)$ and the $J_{2 n}(B ; x)$ are odd functions. So we develop cosine sum approximations for the former and sine sum approximations for the latter. For the cosine approximations, we use as input the values

$$
f_{j}:=J_{n}(B ; j \Delta) \text { or } f_{j}:=J_{n}(j \Delta) \quad j=0, \ldots, 2 m-1, \quad \Delta=\frac{B}{2 m-1},
$$

and for the sine approximations, where we always have $f_{0}=0$ since the function is odd, the values

$$
f_{j}:=J_{n}(B ; j \Delta) \text { or } f_{j}:=J_{n}(j \Delta) \quad j=1, \ldots, 2 m, \quad \Delta=\frac{B}{2 m},
$$

so for both, $2 m$ samples taken at equally spaced points in the interval $[0, B]$. The new approximations are of the so-called Prony-type $[8,6]$.

\subsection{Finite sum cosine approximations}

From $[5,4]$ we obtain the following formulas for the construction of an $m$-term cosine interpolant with coefficients $\alpha_{k}$ and frequencies $\phi_{k}$, satisfying the inter- 
polation conditions

$$
\sum_{k=1}^{m} \alpha_{k} \cos \left(\phi_{k} j \Delta\right)=f_{j}, \quad j=0, \ldots, 2 m-1,
$$

where the sampled (restricted) Bessel function is an even function.

We first solve (9) for the unknown nonlinear parameters $\phi_{k}$ in the interpolant and afterwards for the unknown linear coefficients $\alpha_{k}$.

Let the $\ell \times \ell$ Hankel and Toeplitz matrices $H_{\ell}^{(r)}$ and $T_{\ell}^{(r)}$ be defined by

$$
\begin{aligned}
& H_{\ell, \pm}^{(r)}:=\left(\begin{array}{cccc}
f_{r} & f_{r \pm 1} & \cdots & f_{r \pm(\ell-1)} \\
f_{r \pm 1} & & & \\
\vdots & . \cdot & & \vdots \\
f_{r \pm(\ell-1)} & \cdots & & f_{r \pm(2 \ell-2)}
\end{array}\right) \text {, }
\end{aligned}
$$

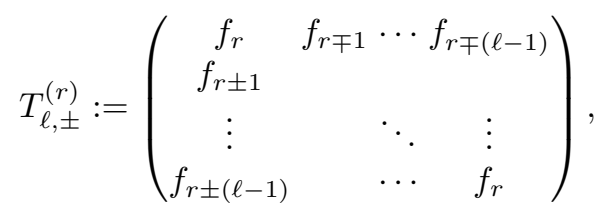

where for $j<0$ formula (4) is used. Because of the uniformity of the sampling at $j \Delta$ and the property

$$
\frac{1}{2} \cos \left(\phi_{k}(j+1) \Delta\right)+\frac{1}{2} \cos \left(\phi_{k}(j-1) \Delta\right)=\cos \left(\phi_{k} \Delta\right) \cos \left(\phi_{k} j \Delta\right)
$$

the generalized eigenvalue problem (11) can be written down for the matrices

$$
\begin{aligned}
C_{m}^{(0)} & :=\frac{1}{4} H_{m,+}^{(0)}+\frac{1}{4} H_{m,-}^{(0)}+\frac{1}{4} T_{m,+}^{(0)}+\frac{1}{4} T_{m,-}^{(0)}, \\
C_{m}^{(1)} & :=\frac{1}{4} H_{m,+}^{(1)}+\frac{1}{4} H_{m,-}^{(1)}+\frac{1}{4} T_{m,+}^{(1)}+\frac{1}{4} T_{m,-}^{(1)} .
\end{aligned}
$$

The values $\cos \left(\phi_{k} \Delta\right), k=1, \ldots, m$ are the generalized eigenvalues of $[5,4]$

$$
C_{m}^{(1)} v_{k}=\cos \left(\phi_{k} \Delta\right) C_{m}^{(0)} v_{k}, \quad k=1, \ldots, m,
$$

where the $v_{k}$ are the generalized eigenvectors. Under the condition that

$$
0 \leq \max _{k} \phi_{k} \Delta \leq \pi
$$

the frequencies $\phi_{k}, k=1, \ldots, m$ can be unambiguously extracted from the generalized eigenvalues $\cos \left(\phi_{k} \Delta\right), k=1, \ldots, m$. With the $\phi_{k}$ identified, the interpolation problem (9) can be solved for the coefficients $\alpha_{k}$. In an exact mathematical setting it suffices to consider a subset of $m$ interpolation conditions of the $2 m$ imposed ones, as the remaining $m$ conditions have become linearly dependent because of the generalized eigenvalue relation (11) satisfied by the $\phi_{k}$. 
With respect to (12) we make the following remarks and observations. With $\Delta=B /(2 m-1)$ condition (12) amounts to

$$
0 \leq \max _{k} \phi_{k} \leq \frac{(2 m-1) \pi}{B},
$$

which implies that the choice of $B$ and $m$ determines which frequencies in $J_{n}(B ; x)$ or $J_{n}(x)$ can be identified without aliasing effect. In other words, the choice of $B$ and $m$ limits the frequency range of the parameters $\phi_{k}$ in the models (9) and (13). In view of the well-known damped cosine asymptotic behaviour [9]

$$
J_{n}(x) \sim \sqrt{\frac{2}{\pi x}} \cos \left(x-\frac{(2 n+1) \pi}{4}\right)
$$

and our own observations formulated in Section 4 , it is reasonable to choose $m$ for a given $B$ such that $B<(2 m-1) \pi$.

\subsection{Finite sum sine approximations}

Also from [4], we find similar formulas for the construction of an $m$-term sine interpolant for $J_{n}(B ; x)$, satisfying

$$
\sum_{k=1}^{m} \alpha_{k} \sin \left(\phi_{k} j \Delta\right)=f_{j}, \quad j=1, \ldots, 2 m,
$$

where the sampled (restricted) Bessel function of the first kind is an odd function. We summarize the method here.

Because the sine analogue of the property (10) is

$$
\frac{1}{2} \sin \left(\phi_{k}(j+1) \Delta\right)+\frac{1}{2} \sin \left(\phi_{k}(j-1) \Delta\right)=\cos \left(\phi_{k} \Delta\right) \sin \left(\phi_{k} j \Delta\right),
$$

we define

$$
\begin{aligned}
S_{m}^{(0)} & :=\frac{1}{2} H_{m,+}^{(1)}+\frac{1}{2} T_{m,+}^{(1)}, \\
S_{m}^{(1)} & :=\frac{1}{4} H_{m,+}^{(2)}+\frac{1}{4} H_{m,+}^{(0)}+\frac{1}{4} T_{m,+}^{(2)}+\frac{1}{4} T_{m,+}^{(0)} .
\end{aligned}
$$

Then the values $\cos \left(\phi_{k} \Delta\right)$ are the generalized eigenvalues of [4]

$$
S_{m}^{(1)} v_{k}=\cos \left(\phi_{k} \Delta\right) S_{m}^{(0)} v_{k}, \quad k=1, \ldots, m .
$$

Under the condition that

or equivalently

$$
\left|\max _{k} \phi_{k} \Delta\right| \leq \frac{\pi}{2}
$$

$$
\max _{k}\left|\phi_{k}\right| \leq \frac{(2 m-1) \pi}{2 B},
$$

the frequencies $\phi_{k}$ can be uniquely extracted from the generalized eigenvalues $\cos \left(\phi_{k} \Delta\right)$ computed from (15) and then the coefficients $\alpha_{k}, k=1, \ldots, m$ can be computed from the interpolation conditions (13) as above. Our recommendation now is to respect $2 B<(2 m-1) \pi$. 


\section{Numerical comparison}

In the following we compare the new Prony-type interpolants, first to partial sums of the Fourier-type series expansion for the functions $J_{n}(B ; x)$ on $[0, B]$ presented in Section 2 and afterwards to the power series and asymptotic series expansions (1) and (2) given in Section 1 . We take $J_{0}(B ; x)$ and $J_{0}(x)$ as example for the cosine approach and $J_{2}(B ; x)$ and $J_{1}(x)$ as example for the sine approach, and such for different values of $B$ and $m$. It is the subject of future work to verify whether our observations for the example functions can be stated for general order $n$, which seems the case from our initial experiments presented in Section 3.3 .

In order to avoid any undesirable effect of rounding errors and make sure that the truncation error incurred by the new approximants is dominant, the computations are performed in the computer algebra environment Maple, using between 800 and 2200 digits, depending on the values of $m$ and $B$.

We use the formula

$$
\frac{\left|J_{n}(B ; x)-R_{m}(x)\right|}{1+\left|J_{n}(B ; x)\right|} \text { or } \frac{\left|J_{n}(x)-R_{m}(x)\right|}{1+\left|J_{n}(x)\right|}
$$

for the relative error from approximating the function value $J_{n}(B ; x)$ or $J_{n}(x)$ by the trigonometric $m$-term sum $R_{m}(x)$. The denominator takes care of any occurrence of zeroes: in their neighbourhood the relative error is gradually replaced by the absolute error.

\subsection{Cosine sum approximants for $J_{0}(B ; x)$}

In Figure 3 and Table 1 we show the $\log _{10}$ relative error of the new approximants introduced in Section 2.2 compared to the Fourier-type partial sums given in Section 2.1, and this for various values of the trigonometric degree $m$ and the interval upperbound $B$. The figures speak for themselves. We do not display results for the even function $J_{1}(B ; x)$ as these are very similar to the ones obtained for $J_{0}(B ; x)$.

Table 1. Maximum $\log _{10}$ relative error of the $m$-th approximants of $J_{0}(B ; x)$.

\begin{tabular}{|c||c|c||c|c||c|c|}
\hline \multicolumn{1}{|c||}{} & \multicolumn{2}{c||}{$B=1$} & \multicolumn{2}{c||}{$B=5$} & \multicolumn{2}{c|}{$B=20$} \\
$m$ & $(6)$ & $(9)$ & $(6)$ & $(9)$ & $(6)$ & $(9)$ \\
\hline 5 & -2.037 & -27.70 & -0.6596 & -13.43 & -0.2729 & -2.637 \\
10 & -2.317 & -66.14 & -0.9248 & -37.88 & -0.3337 & -14.59 \\
25 & -2.702 & -202.5 & -1.305 & -132.3 & -0.2496 & -72.54 \\
50 & -2.999 & -463.1 & -1.601 & -323.0 & -0.4362 & -202.9 \\
75 & -3.173 & -746.2 & -1.775 & -536.2 & -0.5891 & -355.8 \\
100 & -3.298 & -1044 & -1.900 & -764.1 & -0.7055 & -523.5 \\
\hline
\end{tabular}



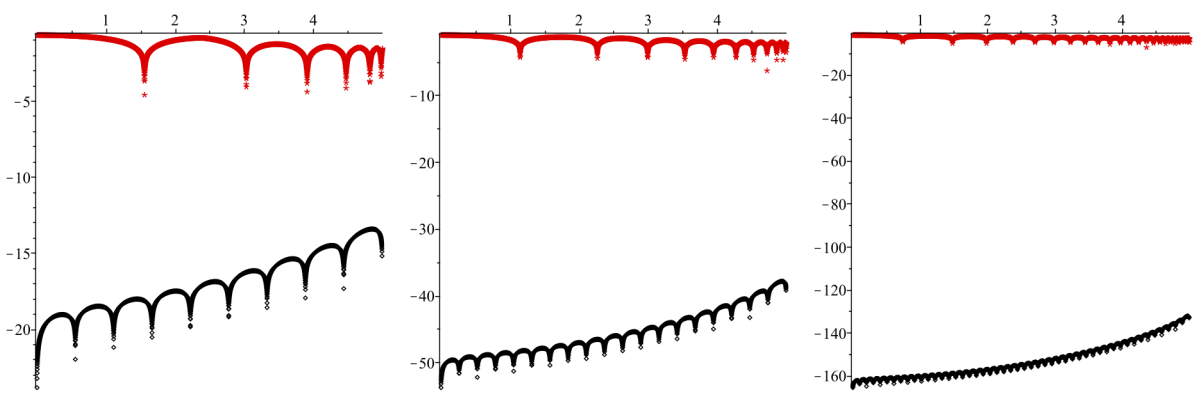

Fig. 3. $\log _{10}$ relative error of the $m$-th Fourier-type (upper red) and cosine Prony-type (lower black) approximants for $m=5,10,25$ (left to right) and $B=5$.

\subsection{Sine sum approximants for $J_{2}(B ; x)$}

In Figure 4 and Table 2 we show the $\log _{10}$ relative error of the new approximants introduced in Section 2.3 compared to the Fourier-type partial sums given in Section 2.1, and this for various values of the trigonometric degree $m$ and the interval upperbound $B$. The figures again speak for themselves. The behaviour is similar when approximating other functions $J_{2 n}(B ; x)$.
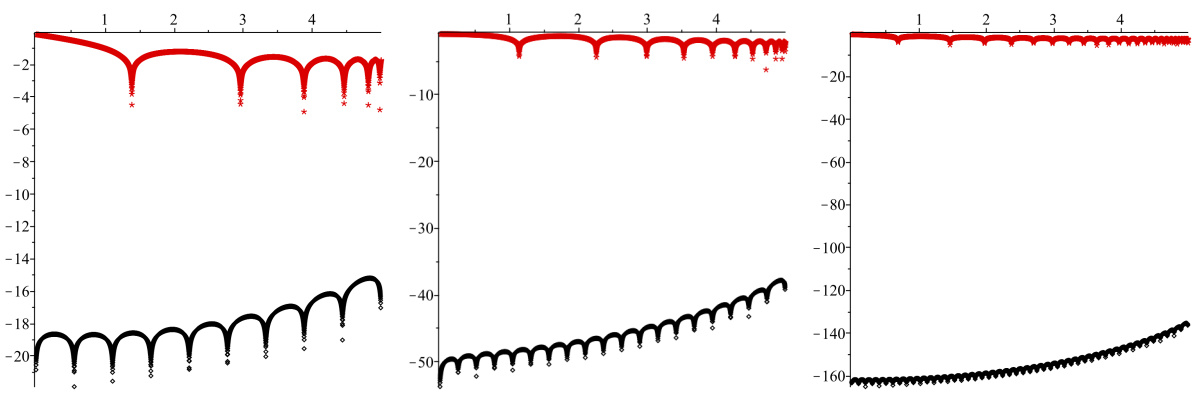

Fig. 4. $\log _{10}$ relative error of the $m$-th Fourier-type (upper red) and sine Prony-type (lower black) approximants for $m=5,10,25$ (left to right) and $B=5$.

\subsection{Power series and asymptotic series sums}

To conclude our numerical illustration, we compare the new Prony-type approximants, now computed for $J_{n}(x)$, to the power series and asymptotic series expansions (1) and (2). In Table 3 we fix $m=20$ but vary $B$ in $[0, B]$ and the order $n$ in $J_{n}(x)$. The cosine and sine sums are, as expected, much better when 
Table 2. Maximum $\log _{10}$ relative error of the $m$-th approximants of $J_{2}(B ; x)$.

\begin{tabular}{|c||c|c||c|c||c|c|}
\hline \multicolumn{1}{|c||}{} & \multicolumn{2}{c||}{$B=1$} & \multicolumn{2}{c||}{$B=5$} & \multicolumn{2}{c|}{$B=20$} \\
$m$ & $(6)$ & $(13)$ & $(6)$ & $(13)$ & $(6)$ & $(13)$ \\
\hline 5 & -1.477 & -30.54 & -0.1408 & -15.22 & -0.1167 & -2.609 \\
10 & -1.615 & -69.63 & -0.2455 & -40.29 & -0.0387 & -15.44 \\
25 & -1.807 & -206.9 & -0.4181 & -163.4 & 0.4884 & -74.29 \\
50 & -1.956 & -468.0 & -0.5604 & -326.8 & 0.5256 & -205.2 \\
75 & -2.044 & -751.5 & -0.6458 & -540.4 & 0.4867 & -358.6 \\
100 & -2.107 & -1049 & -0.7070 & -768.6 & 0.4461 & -526.5 \\
\hline
\end{tabular}

regarding a wider range for $x$, while the expansions (1) and (2) only perform well for either small or large values of the argument.

Table 3. Rough maximum $\log _{10}$ relative error of the 20-th approximants of $J_{n}(x)$ for various $B$ and $n$.

\begin{tabular}{|c||c|c|c||c|c|c||c|c|c|}
\hline \multicolumn{1}{|c||}{$B$} & \multicolumn{3}{c||}{$(1)$} & \multicolumn{3}{c||}{$(2)$} & \multicolumn{3}{c|}{$(9)$ or $(13)$} \\
\hline$n$ & 0.01 & 15 & 100 & 0.01 & 15 & 100 & 0.01 & 15 & 100 \\
\hline 0 & -137 & -1 & 32 & 117 & -13 & -49 & -321 & -56 & -19 \\
\hline 1 & -138 & -3 & 32 & 120 & -14 & -48 & -324 & -83 & $-12^{*}$ \\
\hline 2 & -139 & -3 & 33 & 122 & -13 & -49 & -324 & -82 & -13 \\
\hline 5 & -141 & -5 & 33 & 131 & -13 & -48 & -327 & -70 & $-15^{*}$ \\
\hline 20 & -148 & -13 & 36 & 186 & -8 & -45 & -333 & -101 & -19 \\
\hline
\end{tabular}

The choice for $B$ satisfies the recommendations formulated in the dicussion of (12) and (16), except for the results in Table 3 marked with *. When using $2 B \geq(2 m-1) \pi$ in the sine model, then the frequency range for the $\phi_{k}$ is reduced and introduces an unwanted aliasing effect from the frequencies in the range $[(2 m-1) \pi /(2 B), 1]$.

\section{Simplified procedure}

When inspecting the computed frequencies $\phi_{k}$ in the models (9) and (13) of the previous section, we observe that:

- all (or almost all) the $\phi_{k}, k=1, \ldots, m$ are real and lie in the interval $[0,1]$;

- their distribution becomes denser towards the interval endpoint 1.

In Figure 5 we display a typical situation. 


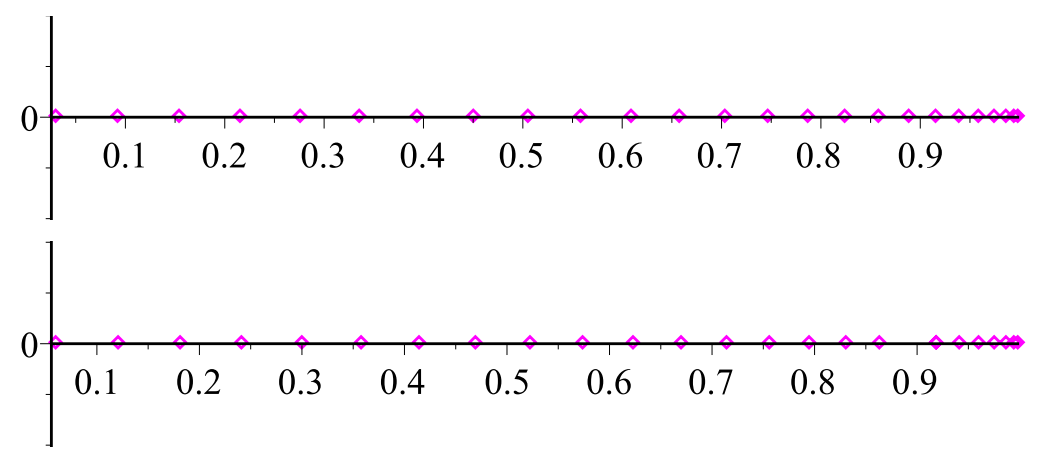

Fig. 5. Distribution of the $\phi_{1}, \ldots, \phi_{m}$ computed for $J_{0}(x)$ (top) and $J_{1}(x)$ (bottom) on $[0, B]$ with $m=25$ and $B=5$.

This behaviour is reminiscent of the behaviour of Chebyshev zeroes and extrema, when restricting them to the interval $[0,1]$. We also note that the frequencies appearing in the simple models (3) coincide with the extrema of a Chebyshev polynomial of the first kind restricted to $[0,1]$. For instance:

- The cosine frequencies $0,1 / 2, \sqrt{3} / 2,1$ in the model for $J_{0}(x)$ are the nonnegative extrema $\cos (3 \pi / 6), \cos (2 \pi / 6), \cos (\pi / 6), 1$ of the Chebyshev polynomial $T_{6}(x)$.

- And the cosine frequencies $0, \sqrt{2} / 2,1$ in the approximation for $J_{4}(x)$ are the non-negative extrema $\cos (2 \pi / 4), \cos (\pi / 4), 1$ of the Chebyshev polynomial $T_{4}(x)$.

Therefore we now investigate the accuracy of the simplified approximants

$$
\sum_{k=1}^{m} \alpha_{k} \cos \left(\tilde{\phi}_{k} x\right)
$$

and

$$
\sum_{k=1}^{m} \alpha_{k} \sin \left(\tilde{\phi}_{k} x\right)
$$

where the $\tilde{\phi}_{k}$ are not obtained from the solution of the generalized eigenvalue problems (11) or (15) but are fixed a priori, preferably as some Chebyshev extrema or zeroes. The coefficients $\alpha_{k}$ are still computed from the system of interpolation conditions (9) or (13), now without the frequencies satisfying a related generalized eigenvalue problem. This system consists of $2 m$ interpolation conditions to fix only $m$ coefficients $\alpha_{k}$. It can be solved in the least squares sense or we can select a square subsystem by disregarding in (7) or (8) the samples taken at odd multiples of $\Delta$. In our experiments we have chosen the latter option.

For the $\tilde{\phi}_{k}$ we consider 5 different schemes, where $T_{n}(x)$ and $U_{n}(x)$ respectively denote the Chebyshev polynomials of the first and second kind: 
1. from the zeroes of $T_{2 m}(x)$ :

$$
\tilde{\phi}_{k}=\cos \left(\frac{(2 k-1) \pi}{4 m}\right), \quad k=1, \ldots, m,
$$

2. from the zeroes of $U_{2 m}(x)$ :

$$
\tilde{\phi}_{k}=\cos \left(\frac{k \pi}{2 m+1}\right), \quad k=1, \ldots, m,
$$

3. from the zeroes of $T_{2 m+1}(x)$ :

$$
\tilde{\phi}_{k}=\cos \left(\frac{(2 k-1) \pi}{2(2 m+1)}\right), \quad k=1, \ldots, m,
$$

4. from the extrema of $T_{2 m}(x)$ :

$$
\tilde{\phi}_{k}=\cos \left(\frac{k \pi}{2 m}\right), \quad k=1, \ldots, m,
$$

5. from the extrema of $T_{2(m-1)}(x)$ :

$$
\tilde{\phi}_{k}=\cos \left(\frac{k \pi}{2(m-1)}\right), \quad k=0, \ldots, m-1 .
$$

In the Tables 4 and 5 we show the maximum relative error of different $m$-term cosine and sine approximations for the functions $J_{0}(x)$ and $J_{1}(x)$ on the interval $[0,20]$ respectively. We compare all $\tilde{\phi}_{k}$ selections for the cosine approximants and all but the last one for the sine approximants (in the current choice, the latter leads to a singular linear system for the $\alpha_{k}$ in the sine model). For completeness we also list the maximum relative error of the Prony-type approximant (9) or (13), which is the unrivalled option. In Table 4 the simplified approximants using frequencies $\tilde{\phi}_{k}$ from either (17), (19) or (21) deliver the smallest truncation errors and follow best the optimum trend of (9), explored in Section 3. The conclusion for Table 5 is similar, now with (17) and (19) for (13).

Table 4. Maximum $\log _{10}$ relative error of the $m$-term cosine approximant for $J_{0}(x)$ on $[0,20]$.

\begin{tabular}{|c||c|c|c|c|c|}
\hline$m$ & 10 & 25 & 50 & 75 & 100 \\
\hline$(9)$ & -14.59 & -72.54 & -202.9 & -355.8 & -523.5 \\
\hline$(17)$ & -8.940 & -58.85 & -176.1 & -316.2 & -471.0 \\
$(18)$ & -2.862 & -21.50 & -71.22 & -132.5 & -201.3 \\
$(19)$ & -7.855 & -56.91 & -173.6 & -313.3 & -467.8 \\
$(20)$ & -1.364 & -21.32 & -71.05 & -133.1 & -201.2 \\
$(21)$ & -6.788 & -54.99 & -171.0 & -310.4 & -464.6 \\
\hline
\end{tabular}


Table 5. Maximum $\log _{10}$ relative error of the $m$-term sine approximant for $J_{1}(x)$ on $[0,20]$.

\begin{tabular}{|c||c|c|c|c|c|}
\hline$m$ & 10 & 25 & 50 & 75 & 100 \\
\hline$(13)$ & -16.24 & -75.10 & -206.0 & -359.4 & -527.3 \\
\hline$(17)$ & -8.759 & -58.21 & -175.2 & -315.0 & -469.7 \\
$(18)$ & -2.825 & -21.99 & -72.02 & -133.5 & -202.4 \\
$(19)$ & -7.723 & -56.28 & -172.6 & -312.1 & -466.5 \\
$(20)$ & -3.149 & -22.29 & -72.31 & -133.8 & -202.7 \\
\hline
\end{tabular}

To construct a trigonometric approximation for a particular $J_{n}(x)$ on a specific interval and guaranteeing a predetermined accuracy, it suffices to choose the appropriate $m$ and a suitable scheme for the $\tilde{\phi}_{k}$ (or computing the $\phi_{k}$ as detailed in Section 2). To this end, truncation error upperbounds for each of the trigonometric approximation schemes (9) and (13), in combination with (17)(21), would be very helpful.

\section{Conclusion}

The proposed Prony-like method generates quite high accuracy approximants, which we believe are unexplored so far. In view of the many physics and engineering applications involving Bessel functions of the first kind of integer order, these exploratory results may offer interesting opportunities.

In the future, a more complete comparison of the newly introduced trigonometric approximants with different representations of the Bessel functions of the first kind, should also be conducted.

Precise statements for general order functions $J_{n}(x)$ on a guaranteed truncation error bound for these interpolants in the interval $[0, B]$ and the exact behaviour of the frequencies present in the oscillating and decaying functions $J_{n}(x)$ form interesting topics for future research.

\section{References}

1. Andrusyk, A.: Infinite series representations for Bessel functions of the first kind of integer order. Tech. rep., Institute for Condensed Matter Physics, Lviv, Ukraine (2012), arXiv preprint arXiv:1210.2109

2. Blachman, N.M., Mousavinezhad, S.H.: Trigonometric approximations for Bessel functions. IEEE Trans. Aerosp. Electron. Syst. AES-22, 2-7 (1986)

3. Cuyt, A., Brevik Petersen, V., Verdonk, B., Waadeland, H., Jones, W.B.: Handbook of Continued Fractions for Special Functions. Springer, Berlin (2008)

4. Cuyt, A., Lee, W.s.: Sparse trigonometric and sinc spectral analysis. Tech. rep., Universiteit Antwerpen (2019), in preparation

5. Giesbrecht, M., Labahn, G., Lee, W.s.: Symbolic-numeric sparse polynomial interpolation in Chebyshev basis and trigonometric interpolation. In: Proc. Workshop on Computer Algebra in Scientific Computation (CASC). pp. 195-204 (2004) 
6. Hildebrand, F.B.: Introduction to numerical analysis. Dover Publications, Inc., second edn. (1987)

7. Oldham, K., Myland, J., Spanier, J.: An Atlas of Functions. Springer, New York, NY, second edn. (2009). https://doi.org/10.1007/978-0-387-48807-3

8. de Prony, R.: Essai expérimental et analytique sur les lois de la dilatabilité des fluides élastiques et sur celles de la force expansive de la vapeur de l'eau et de la vapeur de l'alkool, à différentes températures. J. Ec. Poly. 1, 24-76 (1795)

9. Sneddon, I.N.: The use of integral transforms. Mc-Graw Hill, New York (1972) 